\title{
Energy Deposition in Iron Pentacarbonyl Ions Undergoing Surface-Induced Dissociation in a Fourier Transform Mass Spectrometer
}

\author{
John A. Castoro, Paul V. Rucker*, and Charles L. Wilkins \\ Department of Chemistry, University of California, Riverside, California, USA
}

\begin{abstract}
Internal energy deposition into iron pentacarbonyl positive ions undergoing surface-induced dissociation (SID) in a Fourier transform mass spectrometer is estimated from the abundances and known critical energies of the product fragment ions. A narrow energy distribution, comparable to that reported in earlier BQ and tandem quadrupole SID studies of the same compound, is observed. As judged by the ratio of fragment ions to incident parent ions observed, SID of iron pentacarbonyl in the $3 \mathrm{~T}$ Fourier transform mass spectrometer is more efficient, but results in lower conversion of laboratory to internal energy. This may be a result of the more shallow collision incidence angle employed in the Fourier transform mass spectrometer measurements (a few degrees), which contrasts with the $32-60^{\circ}$ collision angles used in the earlier BQ and tandem quadrupole mass spectrometry studies. Collision-induced dissociation with $\mathrm{He}$ under single collision conditions is also reported. Not unexpectedly, conversion of kinetic to internal energy was lower than found in a previous Fourier transform mass spectrometer study of the iron pentacarbonyl cation employing argon as collision gas under multiple collision conditions. (J Am Soc Mass Spectrom 1992, 3, 445-450)
\end{abstract}

$\mathrm{I}$ $t$ is known that laser desorption (LD), particularly near the desorption threshold (as appears to be optimal for matrix-assisted LD) [1-4] often yields abundant molecular ions and relatively few fragment ions. For structure elucidation, fragment ions are required. In low mass applications (i.e., below a few thousand units) collision-induced dissociation (CID) is often used as the ion activation method of choice for tandem mass spectrometry (MS/MS) studies. As Sheil and Derrick [5] observed in a recent paper, center of mass collision energies decrease significantly as the disparity between mass of collision gas and target molecules grows. Thus, for singly charged higher mass ions, collisions with typical target gases (e.g, He or Ar) do not impart sufficient internal energy to cause fragmentation. For example, collision of $5 \mathrm{keV}$ bovine insulin ions (MW 5774) with helium target gas would produce a relative collision energy in the center of mass frame of only $3.5 \mathrm{eV}$ [6]. This fact, together with the increased number of degrees of freedom present in higher mass organic ions, which may allow energy to be efficiently dissipated before a dissociation limit is reached, leads to the conclusion that

\footnotetext{
* University of California President's Undergraduate Fellow for 1991-1992.

Address reprint requests to Professor Charles L. Wilkins, Department of Chemistry, University of California-Riverside, Riverside, CA 92521 .
}

alternative ion activation methods are required for high mass applications involving singly charged ions such as those typically produced by LD. In Fourier transform mass spectrometry (FTMS) the problem is exacerbated because the translational energy ions may acquire is strictly limited, due to the fact that above a specific energy, which is a function of the cell dimensions and magnetic field strength, they will be ejected. This maximum energy in the $x-y$ (cyclotron motion) plane is inversely proportional to mass and directly proportional to magnetic field strength. Thus, as mass increases, CID becomes less and less efficient. These circumstances prompt the present interest in alternative high mass analysis methodologies, which may offer enhanced analytical performance for applications utilizing an FTMS mass analyzer.

Obvious alternatives for ion activation include photodissociation (PD) and surface-induced dissociation (SID), which was introduced by Cooks and co-workers $[7,8]$ for the analysis of polyatomic molecules in 1985 and is the subject of a recent comprehensive review. From the standpoint of generality, SID seems preferable in that it is readily implemented with most common types of mass analyzers and does not require the presence of a chromophore, which is a necessary prerequisite for $\mathrm{PD}$. Cooks and co-workers succinctly described the rationale for SID as an alternative to CID [7] and subsequently elaborated upon that theme 
in detailed internal energy distribution studies of SID $[9,10]$. Among the disadvantages of CID they noted are: (1) in CID internal energy deposited covers a broad range (although the average value can be controlled through selection of the scattering angle and/or collision energy); and (2) introduction of collision gas into the instrument degrades performance, particularly resolution. The second problem is particularly important for FTMS applications, where low analyzer pressures are mandatory for high resolution measurements.

As mentioned above, application of SID is straightforward, simply requiring that the analyte species be accelerated into a metal target, resulting in relatively efficient fragmentation (judged by yield of fragment ions) followed by mass analysis of the product ions. For SID energies of a few hundred electronvolts and lower this procedure results in deposition of a narrower range of internal energies, with somewhat higher average energy deposition, than achieved by CID. SID has been demonstrated with tandem magnetic quadrupole (BQ) [7], tandem time-of-flight [11], tandem quadrupole [12], and tandem Wien [13] mass spectrometers. More recently, SID also has been adapted for FTMS $[14,15]$, which is performed by using the type of instrument employed in the present study. The advantages of FTMS are well known and include its ability to trap ions resulting from (for example) an LD event, with subsequent PD, SID, CID, or combinations thereof [16] as desired, with moderate to high mass resolution. To perform SID in a Fourier transform mass spectrometer, the selected ionic species is translationally excited by an initial on-resonance excitation, as in CID, but then is accelerated into a cell trap plate for SID [14]. As with CID, the maximum kinetic energies of selected ions are still limited by the cell dimensions and magnetic field strength but, in SID, the fraction of laboratory frame kinetic energy converted to internal energy is increased. Mass resolution is also enhanced over CID due to the absence of collision gas. For example, in the present study, the measured mass resolution for iron pentacarbonyl molecular ion under CID conditions in the 7 Tesla Fourier transform mass spectrometer is 1360 , while the corresponding resolution for the molecular ion in the 3 Tesla Fourier transform mass spectrometer under SID conditions is 1140, corresponding to a resolution of 2600 (roughly twice that obtained in the CID measurements) in a 7 Tesla field.

In this article a study of SID energy deposition in iron pentacarbonyl ions is reported. A primary purpose is to examine the behavior of this well-characterized system under the conditions used in FTMS to compare with a similar earlier study that employed a much different collision angle and mass analyzer [10]. Important issues explored include whether a similar narrow internal energy distribution is observed and whether similar efficiencies are obtained. For the purpose of the present discussion, SID eff- ciency will be defined as the total abundance of mass analyzed fragments divided by the incident ion abundance, $\Sigma F_{1} / \mathbf{P}_{0}$, the "strict definition" in the Cooks" terminology [8]. As in the earlier study, average internal energies are estimated by exploiting the known critical energies for loss of successive carbonyl groups [17]. Energy transfer resulting from the inelastic surface collision is estimated by dividing the relative abundances of fragment ions by the known critical energy intervals for the iron pentacarbonyl fragments (eq 1).

$$
\mathbf{P}(\epsilon)=\frac{\left[\mathrm{F}_{\mathbf{n}}\right]^{+}}{\mathbf{E}_{\mathbf{0}}(\mathbf{n}+\mathbf{1})-\mathbf{E}_{0}(\mathbf{n})}
$$

This value approximates the fraction of the parent ions containing internal energies between the critical energy, $E_{0}(n)$, of fragment $F_{n}$ and the energy, $E_{0}(n+$ $1)$, of fragment $F_{n+1}$. The total energy deposition can be estimated from the product ion spectrum. It is also of interest to compare results of SID with those of CID of the same compound under FTMS conditions.

\section{Experimental}

Two different Fourier transform mass spectrometers were utilized in this study. SID experiments were performed by using a $3 \mathrm{~T}$ Fourier transform mass spectrometer-1000 (Nicolet Analytical Instruments, Madison, WI) upgraded with a standard Fourier transform mass spectrometer-2000 dual cell $(4.76 \mathrm{~cm}$ cubic cells) chamber assembly and manual direct insertion probe. CID experiments were performed by using a $7 \mathrm{~T}$ Nicolet Fourier transform mass spectrometer-2000 $4.76 \mathrm{~cm}$ cubic dual cell instrument.

Iron pentacarbonyl (Aldrich Chemical Co., Inc., Milwaukee, WI) was introduced through the batch gas inlet to a source pressure of $5 \times 10^{-7}$ torr, resulting in a pressure of $\sim 6 \times 10^{-8}$ torr in the analyzer region. Twelve electronvolts electron ionization was used to minimize fragmentation. Ions were formed in the source cell and transferred to the analyzer cell through the $2-\mathrm{mm}$ diameter conductance limit. For SID or CID, lower mass ion fragments were ejected by multiple ejection sweeps. For each experiment cyclotron motion of the chosen parent ion was excited by an attenuated on-resonance cyclotron frequency excitation. By varying the attenuation and duration of the excitation radiofrequency ions can be excited to a calculated radius and translational energy. After applying this excitation, for SID, a $+25 \mathrm{~V}$ pulse was applied to the analyzer rear trap plate, repelling the positively trapped ions and causing them to spiral into the conductance limit separating the source and analyzer cells and to fragment. The conductance limit and analyzer trap plate were maintained at $3 \mathrm{~V}$ throughout the experiment, except during the $+25 \mathrm{~V}$ analyzer plate acceleration event. The acceleration du- 
ration was set to 1.5 times the calculated flight time (typically, in the range of 40-50 $\mu \mathrm{sec}$ ) (SIMION V4.0, Idaho National Engineering Laboratory, EG\& G Idaho, Inc., Idaho Falls, ID) of the parent ion from the center of the cell to the conductance limit. This is to allow for single collisions of a large percentage of ions trapped and to provide a deceleration field for the daughter ions produced. After a 10-ms delay the daughter ions were excited by a broadband frequency sweep and detected.

Equation 2 [18] was used to estimate the cyclotron translational energy

$$
K E_{x, y}=\frac{q V_{r f}^{2} t^{2}}{8 d^{2} m}
$$

where, $\mathbf{K E}_{\boldsymbol{x}, \boldsymbol{y}}=$ kinetic energy in electronvolts, $\mathbf{q}=$ ion charge, coulombs, $V=$ one half the peak-to-peak radiofrequency voltage of the excitation plates, volts, $\mathbf{t}=$ duration of the excitation, seconds, $\mathbf{d}=$ distance between the excitation plates, meters, $\mathbf{m}=$ ion mass, kilograms.

Because it is known that, for a cubic cell, eq 2 overestimates the average kinetic energy by a factor of approximately 93\% [19], these calculated values should be adjusted by dividing them by 1.93 to obtain more appropriate estimates. The average $z$-axis translational energy of the ions at impact is independent of mass and calculated to be approximately $1 \mathrm{eV}$ (SIMION V4.0, Idaho National Engineering Laboratory, EG\&G Idaho, Inc., Idaho Falls, ID). SID collision energy is therefore selected by varying the cyclotron translational energy. The resulting net kinetic energy is the vector sum of the cyclotron and $z$-axis kinetic energies, ignoring the negligible initial thermal energy at room temperature [14].

For CID experiments, a static pressure of $1 \times 10^{-6}$ torr of helium collision gas was used, with a 3-ms CID time following translational excitation. Again, translational kinetic energy was estimated by using eq 2 . Typically, 3000 time-domain data sets for SID experiments and 100 for CID experiments were co-added and subjected to Fourier transformation to obtain frequency domain spectra.

\section{Results and Discussion}

Collision of polyatomic ions with a surface, resulting in SID, is a two-stage process. Initially, ion activation occurs during the surface interaction time (estimated to be on the order of $10^{-14} \mathrm{~s}$ ) [8]. Unimolecular dissociation then follows at a much slower rate, with maximum rate constants on the order of $10^{10}-10^{12}$ $\mathrm{s}^{-1}$. Thus, FIMS, with its trapping ability and inherently high mass resolution, should be especially well suited for SID analysis. For the experiments reported here, the 10-ms delay between the collision events and measurement of reflected elastically scattered par- ent ions and fragments resulting from the inelastic collisions permits $10^{7}$ to $10^{9}$ decompositions to occur, ample for FTMS detection. Furthermore, as previously shown [14], the ability to couple SID with LD in the Fourier transform mass spectrometer permits application of SID to structure elucidation of high mass nonvolatiles with higher mass resolution than previously demonstrated. The present study and the prior quantitative SID-FTMS study establish that efficiency is significantly better than reported in the prior studies using different types of mass analyzers. Even employing the "strict" definition of efficiency mentioned above, FTMS efficiencies are in the same range or higher than those defined by using the more liberal definition of efficiency that compensates for second stage mass analysis losses by substituting $\mathrm{P}_{i}^{\prime}$, the transmitted precursor ion abundance in the absence of SID (typically much lower than $P_{0}$ ) for $P_{0}$. For example, efficiencies using the latter definition are typically $2-15 \%$ (see ref 8 , Table 4 ), while FTMS efficiencies range from about $1-30 \%$ by using the more demanding "strict" definition. For example, in the present study, $35 \mathrm{eV}$ SID of iron pentacarbonyl is $3.8 \%$ efficient.

Figure 1 shows results of SID of $\mathrm{Fe}(\mathrm{CO})_{5}^{+}$using three different collision energies, ranging from 35 to $141 \mathrm{eV}$. As expected, at lower collisional energies the lower critical energy products dominate. As collision energy is increased, higher critical energy product ions are observed. Figure 2 is a plot of internal energy distributions for iron pentacarbonyl ions at four different SID cnergies. Each point in the plot represents the relative abundances of a selected mass fragment between two critical energies. The critical energies are shown along the $x$-axis. For example, the fraction of parent ions having an internal energy corresponding to that required to form the $\mathrm{Fe}(\mathrm{CO})_{3}^{+}$fragment can be approximated by dividing its relative abundance by the critical energy difference between $\mathrm{Fe}(\mathrm{CO})_{3}^{+}$and $\mathrm{Fe}(\mathrm{CO})_{2}^{+}$. Similarly, the fraction of parent ions having the requisite energy to produce $\mathrm{Fe}^{+}$is approximated by dividing its relative abundance by the difference in critical energies of $\mathrm{Fe}^{+}$and $\mathrm{FeC}^{+}$. Importantly, the narrow internal energy distributions characteristic of subkiloelectronvolt SID of polyatomics previously reported [9] is maintained. Peak widths of the iron pentacarbonyl distributiuns at half maximum are 3.3 $\mathrm{eV}, 4.1 \mathrm{eV}$, and $6.8 \mathrm{eV}$, corresponding to $35 \mathrm{eV}, 72$ $\mathrm{eV}$, and $141 \mathrm{eV} \mathrm{SID,} \mathrm{respectively} \mathrm{(see} \mathrm{Figure} \mathrm{2).} \mathrm{As}$ summarized in Table 1, the average conversion of laboratory to internal energy ranges from about $7.1 \%$ for $35 \mathrm{eV}$ collisions down to $2.8 \%$ for $228 \mathrm{eV}$ collisions, with average internal energies estimated to be between 2.5 and $6.3 \mathrm{eV}$, respectively. For comparison, Figure 3 shows the $72 \mathrm{eV}$ SID spectrum obtained by FTMS compared with the $60 \mathrm{eV}$ SID spectrum measured by using a $B Q$ mass spectrometer [10]. It is clear that the FTMS SID experiment results in a lower fraction of internal energy deposition (approximately 

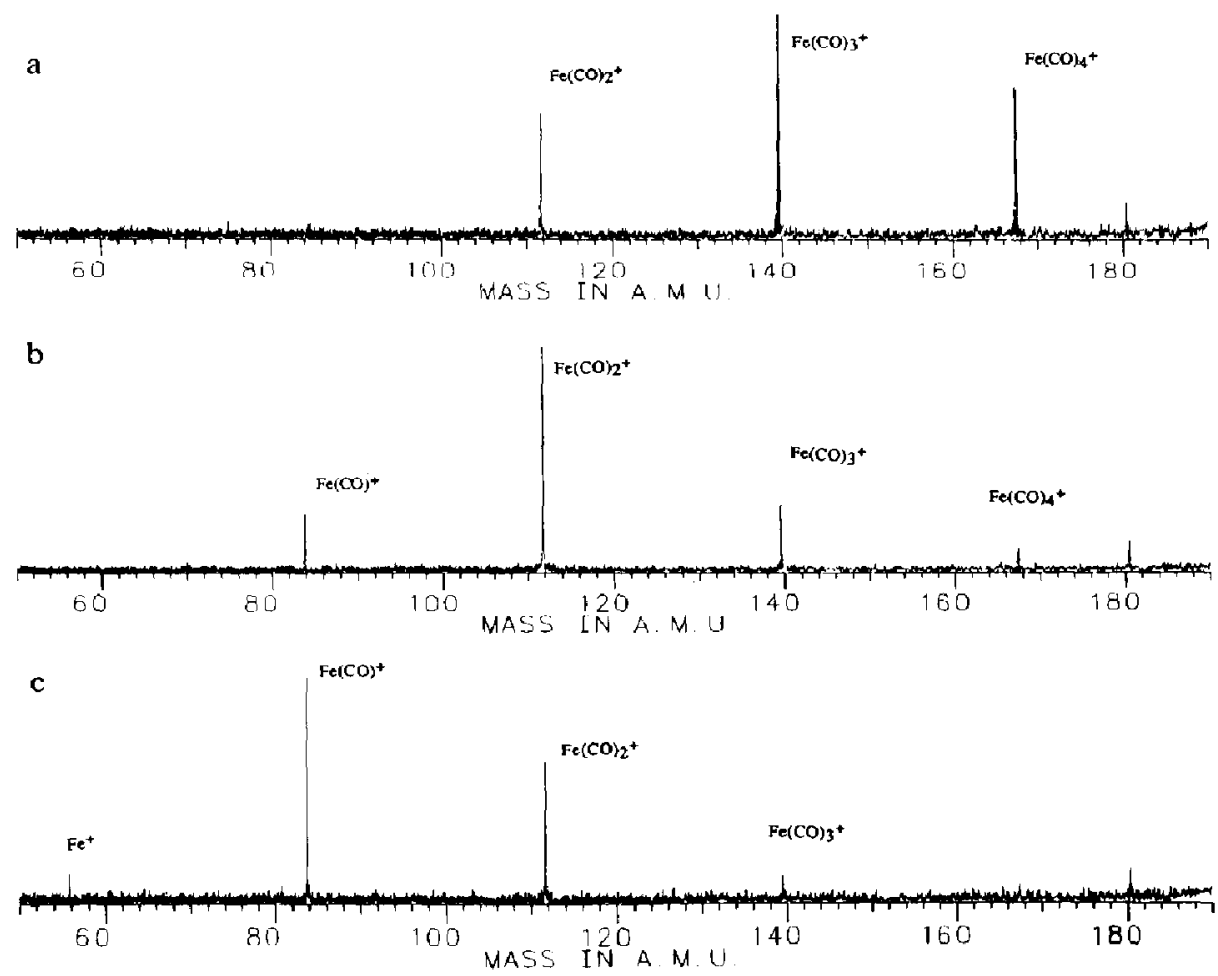

Figure 1. Elcetron ionization SID $3 \mathrm{~T}$ Fouricr transform mass spectra of $\mathrm{Fe}(\mathrm{CO})_{5}^{+}$obtained with three different collision energies: (a) $35 \mathrm{eV}$; (b) $72 \mathrm{eV}$; (c) $141 \mathrm{eV}$.

$6 \%$ of the collision energy versus about $11 \%$ for the $\mathrm{BQ}$ instrument measurement reported in ref 10 ). This is not unexpected considering that the angle of incidence in the FTMS experiment is only a few degrees,

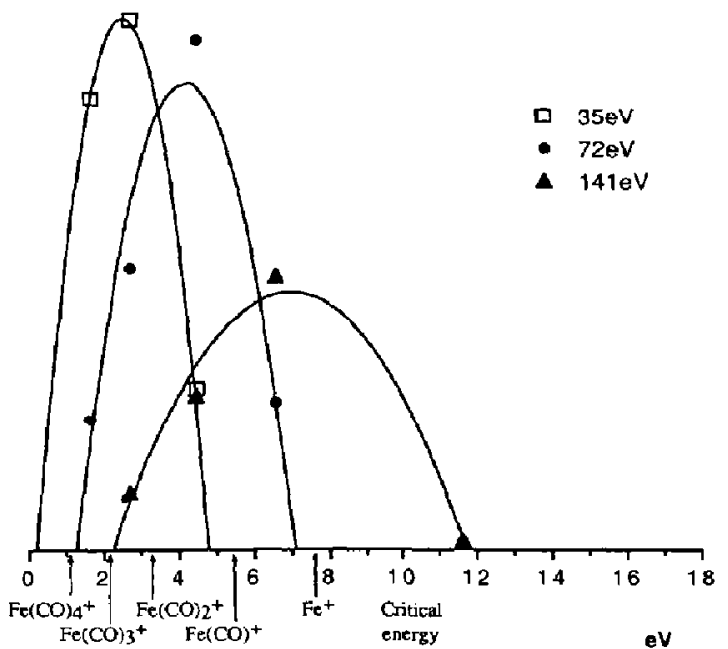

Figure 2. Internal energy distributions of activated parent ions obtained by SID of $\mathrm{Fe}(\mathrm{CO})_{5}^{+}$at three energies: $\square 35 \mathrm{eV}$; 72 $\mathrm{eV} ; \sim 141 \mathrm{eV}$. compared with the $32^{\circ}$ collision angle used in the $\mathrm{BQ}$ experiments and incidence angles between 45 and $60^{\circ}$ mentioned in the triple quadrupole SID study of several organic compounds [11]. A plot of the estimated internal energy distributions for CID of iron pentacarbonyl with $\mathrm{He}$ at three collision energies by FTMS is shown in Figure 4. The average internal energy deposition increases with collisional energy but the fractional conversion decreases very rapidly. For example, the high energy fragment, $\mathrm{Fe}^{+}$, is not observed at the higher CID energy under the single collision conditions used but it is produced by $141 \mathrm{eV}$ SID (Figure 5). However, CID of iron pentacarbonyl in a Fourier transform mass spectrometer under multiple collision conditions, using argon as the collision gas, resulted in almost complete fragmentation to $\mathrm{Fe}^{+}$at collision energies as low as $100 \mathrm{eV}$ [20]. Thus, for this compound conversion of laboratory to internal energy as a result of low energy gas-phase collisions can be very efficient when a high cross-section collision gas and multiple collision conditions are employed.

\section{Conclusions}

SID of iron pentacarbonyl under FTMS conditions results in a lower conversion of laboratory to internal energy than reported in the previous SID study, which 
Table 1. Comparison of average internal energy distributions obtained by SID and CID of iron pentacarbonyl ions using Fourier transform and tandem magnetic-quadrupole mass spectrometers

\begin{tabular}{ccccc}
\hline Instrument and method & $\begin{array}{c}\text { Laboratary kinetic } \\
\text { energy (eV) }\end{array}$ & $\begin{array}{c}\text { Corrected } \\
\text { energy (ref 20) }\end{array}$ & $\begin{array}{c}\text { Average internal } \\
\text { energy (eV) }\end{array}$ & $\begin{array}{c}\text { Energy } \\
\text { conversion (\%) }\end{array}$ \\
\hline FTMS, SID & 67 & 35 & 2.5 & 7.1 \\
& 138 & 72 & 4.0 & 5.6 \\
& 273 & 141 & 5.6 & 4.0 \\
& 440 & 228 & 6.3 & 2.8 \\
BO, SID & & & & \\
& 40 & - & 5.7 & 14.0 \\
& 60 & - & 6.6 & 11.0 \\
FTMS, CID & 80 & - & 7.0 & 8.8 \\
& 130 & 21 & 7.7 & 5.9 \\
& 40 & 46 & 1.9 & 9.0 \\
& 89 & 82 & 2.3 & 5.0 \\
& 158 & 174 & 2.3 & 2.8 \\
& 336 & 2.6 & 1.5 \\
\hline
\end{tabular}

Equation 2.

${ }^{b}$ Data from ref 10.

employed a BQ mass analyzer. However, a similarly narrow energy distribution was observed and efficiency appears to be significantly better when judged by comparison of fragment ion yields relative to incident parent ion abundances. This is probably a result of the much greater second stage mass analysis efficiency realized in the FTMS experiment. When com-

a

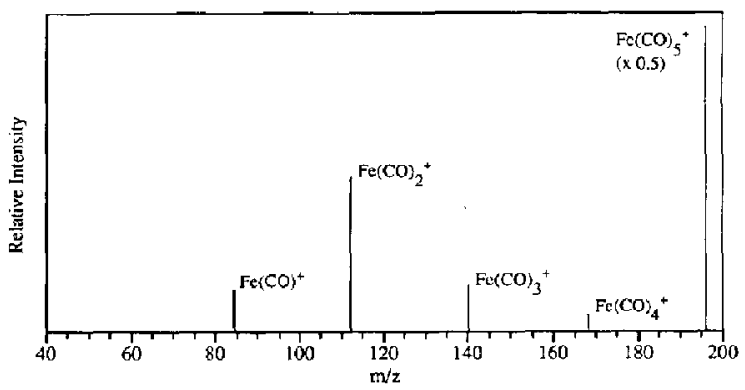

b

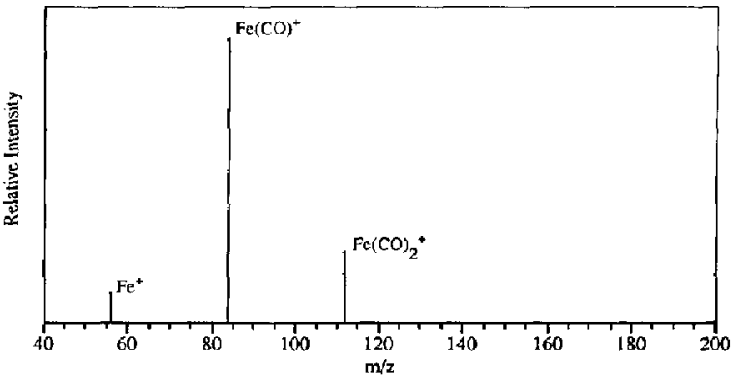

Figure 3. A stick plot comparison of (a) the 3 T FTMS SID mass spectrum of $\mathrm{Fe}(\mathrm{CO})_{5}^{+}$at $72 \mathrm{eV}$ collision energy with (b) 60 eV SID using a magnetic-quadrupole (BQ) tandem mass spectrometer (ref 10). pared with He CID in a 7 Tesla Fourier transform mass spectrometer, 3 T SID clearly resulted in greater parent ion activation and made possible higher mass resolution. However, neither SID nor He CID resulted in as much activation as reported in the earlier FTMS CID study, utilizing argon as collision gas. This is a consequence of the higher collision cross-section and more favorable center of mass energy of argoniron pentacarbonyl collisions, as well as the multiple collision conditions employed. It is clear that SID has a good deal of promise as a means of activating high mass lašer-desorbed ions for structural analysis in a Fourier transform mass spectrometer. Internal energy deposition is adequate for such analytical purposes,

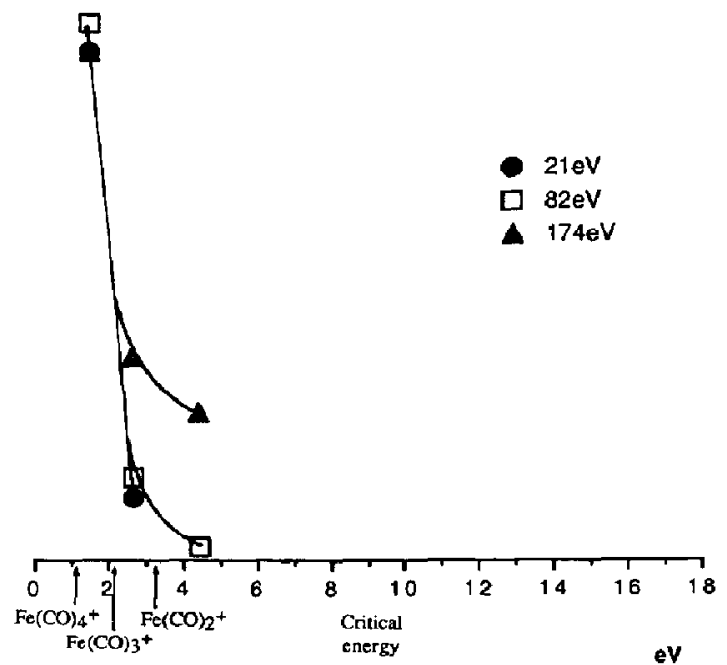

Figure 4. Internal energy distribution of activated parent ions obtained by single collision $\mathrm{He} \mathrm{CID}$ of $\mathrm{Fe}(\mathrm{CO})_{5}^{+}$at three energies: $-21 \mathrm{eV} ; \square 82 \mathrm{eV} ; \wedge 174 \mathrm{eV}$. 

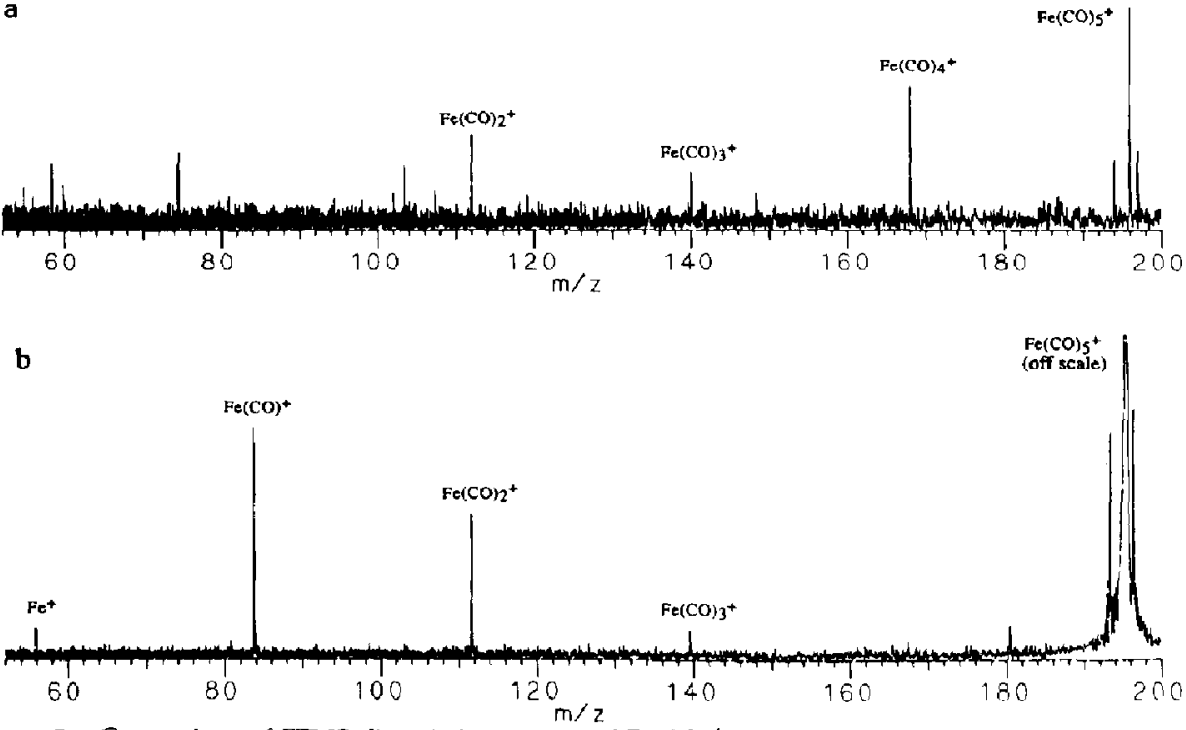

Figure 5. Comparison of FTMS dissociation spectra of $\mathrm{Fe}(\mathrm{CO})_{5}^{+}$by (a) $174 \mathrm{eV} \mathrm{CID}$ with He at 7 Tesla and (b) $141 \mathrm{eV} \mathrm{SID} \mathrm{at} 3$ Tesla.

high mass resolution of product ions should be possible, and greater efficiency could be achieved by optimization of analytical parameters.

\section{Acknowledgments}

We gratefully acknowledge support from the Petroleum Research Fund, administered by the American Chemical Society (grant 23135-AC5). We also gratefully acknowledge the numerous very helpful comments provided by the reviewers of this manuscript.

\section{References}

1. Karas, M.; Bachmann, D.; Bahr, U.; Hillenkamp, F, Int. I. Mass Spectrom. Ion Processes 1987, 78, 53.

2. Karas, M.; Hillenkamp, F. Anal. Chem. 1988, 60, 2299.

3. Hillenkamp, F.; Karas, M.; Ingendoh, A.; Stahl, B. In Biological Mass Spectrometry, Burlingame, A. L.; McCloskey, J. A., Eds.; Elsevier; Amsterdam, 1990; pp 49-60.

4. Tanaka, K.; Waki, H.; Ido, Y.; Yoshida, Y.; Yoshida, T. Rapid Commun. Mass Spectrom. 1988, 2, 151.

5. Sheil, M. M.; Derrick, P. J. Org. Mass Spectrom. 1988, 23, 429.

6. Orlando, R.; Fenselau, C.; Cotter, R. Org. Mass Spectrom. 1989, 24, 1033 .
7. Mabud, M. D. A.; DeKrey, M. J.; Cooks, R. G. Int. J. Mass Spectrom. Ion Processes 1985, 67, 285.

8. Cooks, R. G.; Ast, T.; Mabud, M. D. A. Int. J. Mass Spectrom. Ion Processes 1990, 700, 209.

9. Wysocki, V. H.; Kenttamaa, H. I.; Cooks, R. G. Int J. Mass Spectrom. Lor Processes 1987, 75, 181.

10. DeKrey, M. J.; Kenttamaa, H. I., Wysocki, V. H.; Cooks, R. G. Org. Mass Spectrom. 1986, 21, 193.

11. Schey, K.; Cooks, R. G.; Grix, R.; Wollnik, H. Int. J. Mass Spectrom. Ion Processes 1987, 77, 49.

12. Bier, M. E.; Amy, J. W.; Cooks, R. G.; Syka, J. E. P.; Ceja, P.) Stafford, G. Int. I. Mass Spectrom. Ion Processes 1987, 77, 31.

13. Aberth, W. Anal. Chem. 1990, 62, 609.

14. Ijames, C. F, Wilkins, C. L. Anal. Chem. 1990, 62, 1295.

15. Williams, E. R.; Henry, K. D.; McLafferty, F. W.; Shabanowitz, J.; Hunt, D. F. J. Amer. Soc. Mass Spectrom. 1990, $1,413$.

16. Nuwaysir, L. M.; Casturo, J. A.; Wilkins, C. L. Org. Mass Spectrom. 1991, 26, 721.

17. Kenttamaa, H. I,; Cooks, R. G. Int. I. Mass Spectrom. Ion Processes 1985, 64, 79.

18. Comisarow, M. B. J. Chem. Phys. 1978, 69, 4097.

19. Grosshans, P. B.; Shields, P.; Marshall, A. G. I. Amer. Chem. Soc. 1990, 112, 1275.

20. Cody, R. B.; Weil, D. A. Abstracts of the 36th ASMS Conference on Mass Spectrometry ard Allied Topics; San Francisco, CA, June 1988; pp 1332-1333. 